\title{
A Method for Analyzing the Voltage Deviation Isolation Performance With an Application in Two-Stage High-Frequency Isolated AC-DC Converters in LVDC Systems
}

\author{
Yangxin QIU, Qing ZHONG, Yuming ZHAO, Gang WANG, and Longjun WANG
}

\begin{abstract}
The differences between the power quality at the AC and DC sides, caused by AC-DC converters with constant DC voltage control, commonly exist in low-voltage direct current (LVDC) systems. The voltage deviation isolation (VDI) is one of the typical phenomenons. A method is proposed to analyze the VDI performance and applied in two-stage high-frequency isolated AC-DC converters, which can isolate not only the faults but also the voltage deviation at the $\mathrm{AC}$ side and improve the voltage quality at the DC side. First, the voltage deviation isolation ratio (VDIR) is defined to assess VDI. Based on VDIR, the operation characteristics of AC-DC converters with constant DC voltage control are analyzed and the range of VDI is defined. Second, with considering the conditions for stable operation of AC-DC converters, constraints on power transmission limit and static stability limit are used to determine the range of VDI. Third, a simulation model is established and the feasibility and validity of the method are verified. Finally, the main factors affecting the range of VDI are analyzed. Actually, the method proposed is adapted to various types of AC-DC converters with constant DC voltage control.
\end{abstract}

Index Terms - High-frequency isolated AC-DC converter, power transmission limit, static stability limit, voltage deviation isolation (VDI), voltage deviation isolation ratio (VDIR).

\section{INTRODUCTION}

$\mathrm{A}$ $\mathrm{S}$ the worldwide demand for low-carbon emission increases, low-voltage direct current (LVDC) systems develop rapidly due to the convenience to access distributed power supply [1]-[3], which brings new challenges to power quality. Some research has done for power quality of LVDC, such as the definition and measurement of indices [4], analysis and control

Manuscript received June 18, 2021; revised August 5, 2021; accepted August 23, 2021. Date of publication September 30, 2021; date of current version September 18 , 2021. This work was supported in part by the Natural Science Foundation of Guangdong Province, China under Grant 2021A1515012087 and in part by China Southern Power Grid Co., Ltd. China under Grant 090000KK52180116. (Corresponding Author: Qing Zhong.)

Y. Qiu, Q. Zhong, G. Wang, and L. Wang are with the School of Electric Power, South China University of Technology, Guangzhou, China (e-mail: epqzhong@ scut.edu.cn; qyx.scut@qq.com; wangg@scut.edu.cn; epwlj@scut.edu.cn).

Y. Zhao is with Shenzhen Power Supply Bureau Co., Ltd., Shenzhen, China (e-mail: zhaoym98@sina.com).

Digital Object Identifier 10.24295/CPSSTPEA.2021.00020 of LVDC power quality [5]-[8]. For grid-connected LVDC systems, the power quality at the point of common coupling (PCC) also attracts attention [9]. Nevertheless, the existing research usually pays attention to the power quality only at the $\mathrm{AC}$ side [10] or DC side. The studies on the differences between the power quality at the $\mathrm{AC}$ side and $\mathrm{DC}$ side for grid-connected LVDC systems are rare. In grid-connected LVDC systems, ACDC converters usually adopting constant $\mathrm{DC}$ voltage control are used for LVDC systems to connect to the AC grid, which can hold the $\mathrm{DC}$ voltage with different $\mathrm{AC}$ voltage deviations. It is necessary to research the difference between the power quality at the AC side and DC side of the grid-connected converters, which is helpful to understand the defense of LVDC systems against power quality issues from the $\mathrm{AC}$ grid.

When there is a voltage deviation at the AC side, the ACDC converters may be able to keep the DC voltage at the instruction value. The phenomenon that the voltage deviation at the DC side is different from the AC side is called voltage deviation isolation (VDI) in the paper. It is obvious that the performance of VDI of AC-DC converters is an important factor to improve the power quality at the DC side of LVDC systems. In previous studies, voltage deviation, voltage qualification rate and voltage regulation are common indices to applied in voltage deviation research. Voltage deviation and qualification rate, are the indices without comparison features and not suitable for VDI. Voltage regulation is proposed to describe the difference between the conventional no-load voltage and the voltage at load excluding the correcting effect of stabilizing means if any [11]. However, voltage regulation is not suitable for VDI. For first example, the low AC voltage in rural areas caused by the long lines can be transformed into a normal DC voltage by AC/DC converters. In this case, voltage regulation is obviously not suitable without the change of loads. For second example, when the AC voltage changes because of change of the loads of other lines, and the loads of the line connected to the LVDC systems remain the same, voltage regulation is obviously not suitable. Both cases belong to VDI but can not be described by voltage regulation. Therefore, it is necessary to propose a new index and method to assess and analyze VDI.

As one type of the typical grid-connected AC-DC converters, 
two-stage high-frequency isolated AC-DC converters have advantages of efficiency, energy density and fault isolation [12]. Previous research on high-frequency isolated AC-DC converters mainly focus on topology design, control strategy, new materials [13], performance analysis [12] and et al. In [14], a topology is presented for high-frequency AC-DC converters to improve the efficiency and suppress EMI noise. The control strategies are modified to suppress current harmonic, improve efficiency and eliminate the DC bias flux in [9], [15], [16] respectively. However, there is little research to quantitatively analyze the VDI performance for two-stage high-frequency isolated AC-DC converters.

With the above background, a method is proposed to analyze the VDI performance and applied in two-stage high-frequency isolated AC-DC converters in LVDC systems. In section II, the voltage deviation isolation ratio (VDIR) is defined to assess VDI. With considering the operation characteristics of AC-DC converters with constant DC voltage control, the definition of the range of VDI is presented. In section III, based on the conditions for stable operation of AC-DC converters, constraints on the power transmission limit and the static stability limit are considered to determine the range of VDI. The formulas to analytically compute steady-state points rather than to simulate or numerically calculate are given. In section IV, the results of theoretical analysis are testified by the comparison with simulation results. In section $\mathrm{V}$, the main factors affecting the range of VDI are analyzed with the control variable method. The paper is concluded in section VI.

\section{VDIR AND THE RANGE OF VDI}

\section{A. Voltage Deviation Isolation Ratio}

Differences between voltage deviations at both ends of the power conversion module are common, which is called VDI in the paper. To assess VDI, VDIR is defined as:

$$
\operatorname{VDIR}=\left(1-\frac{\Delta U_{2}}{\Delta U_{1}}\right) \times 100 \%
$$

Where $\Delta U_{1}$ and $\Delta U_{2}$ are voltage deviations at both ends of the power conversion module respectively shown in Fig. 1. When $\Delta U_{1}=0$, calculating VDIR is meaningless. VDIR represents the percentage reduction of $\Delta U_{2}$ relative to $\Delta U_{1}$. Therefore, it is noted that $\Delta U_{1}$ should remain the same when a VDIR is compared with another.

VDIR for grid-connected AC-DC converters is as (2):

$$
\operatorname{VDIR}=\left(1-\frac{\Delta U_{\mathrm{DC}}}{\Delta U_{\mathrm{AC}}}\right) \times 100 \%
$$

Where $\Delta U_{\mathrm{DC}}$ and $\Delta U_{\mathrm{AC}}$ are voltage deviations based on DC instruction voltage and $\mathrm{AC}$ rated voltage respectively. VDIR is the percentage reduction of $\Delta U_{\mathrm{DC}}$ relative to $\Delta U_{\mathrm{AC}}$.

\section{B. Range of VDI}

Without consideration of the output limit and deviation

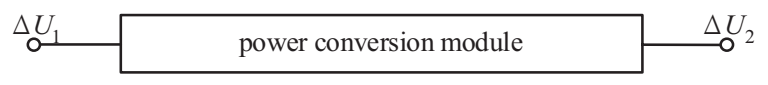

Fig. 1. Voltage deviations at both ends of the power conversion module.

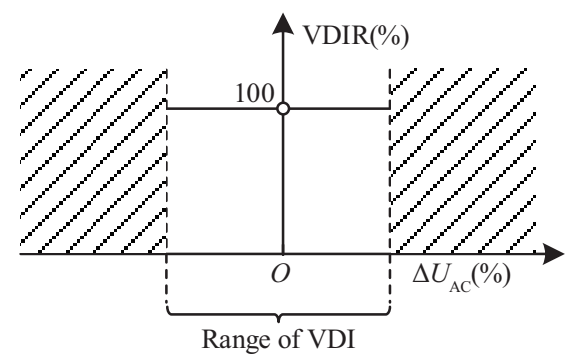

Fig. 2. Schematic diagram of the range of VDI.

of proportional-integral (PI) controller, there are only two operating states for AC-DC converters with constant DC voltage control: Run at the instruction DC voltage so that VDIR $=100 \%$ or $\Delta U_{\mathrm{AC}}=0 \%$, or run at an unstable state.

The range of $\Delta U_{\mathrm{AC}}$ where AC-DC converters can run at the instruction DC voltage is defined as the range of VDI, as shown in Fig. 2.

\section{Determination of the RANGe of VDI For Two- \\ Stage High-Frequency Isolated AC-DC CONVERTERS}

The topology and control strategy of the two-stage highfrequency isolated AC-DC converter in LVDC systems are shown in Fig. 3. To simplify the analysis, the AC grid is regarded as an $\mathrm{AC}$ source in series with an inductor, and only the resistive load is considered at the DC side.

In topology part, $u_{\mathrm{sabc}}$ are the phase $\mathrm{AC}$ grid source voltages. $N$ is the neutral point. $u_{\mathrm{abc}}$ and $i_{\mathrm{abc}}$ are the phase voltages and currents at the PCC respectively. $u_{\text {sabc }}$ are the phase output voltages of the arms of the AC-DC converter. $u_{\mathrm{dc}}$ and $i_{\mathrm{dc}}$ are the voltage and current at the DC side of the front stage of the AC-DC converter respectively. $u_{\mathrm{dcl} l}$ and $i_{\mathrm{dcl}}$ are the voltage and current at the DC side of the back stage of the AC-DC converter. $L_{\mathrm{s}}$ is the equivalent inductance of the $\mathrm{AC}$ grid. $L$ is the inductance of the filter at the $\mathrm{AC}$ side. $C$ and $C_{1}$ are capacitors at the DC side of the front and back stages of the AC-DC converter. $L_{\mathrm{hf}}$ and $R_{\mathrm{hf}}$ are the inductance and equivalent resistance in series with the two-stage high-frequency isolated transformer respectively. $k$ is the rated voltage ratio of the twostage high-frequency isolated transformer. $R_{L}$ is the resistance load.

In control strategy part, $s_{\mathrm{j}}(\mathrm{j}=\mathrm{a}, \mathrm{b}, \mathrm{c}, \mathrm{g}, \mathrm{g} 1)$ are the switching functions representing the switching state of the power electronic devices of the front stage. When the switch of the upper/lower arm is conducted, $s_{\mathrm{j}}(\mathrm{j}=\mathrm{a}, \mathrm{b}, \mathrm{c})$ is equal to $1 /-1$. When the switch corresponding to $\left.s_{\mathrm{j}} \mathrm{j}=\mathrm{g}, \mathrm{g} 1\right)$ is or not conducted, $s_{\mathrm{j}}(\mathrm{j}=$ $\mathrm{g}, \mathrm{g} 1)$ is equal to $1 /-1 . \omega$ and $\omega_{\mathrm{hf}}$ are the rated frequency of $\mathrm{AC}$ grid and high-frequency isolated transformer respectively. $\theta_{\mathrm{pll}}$ is the output of PLL. $\theta$ is the phase difference between $u_{\mathrm{abc}}$ and $u_{\mathrm{sabc}} \cdot u_{\mathrm{dq}}, i_{\mathrm{dq}}$ and $s_{\mathrm{dq}}$ are $u_{\mathrm{abc}}, i_{\mathrm{abc}}$ and $s_{\mathrm{abc}}$ in dq coordinate. $i_{\mathrm{dqree}}$, 


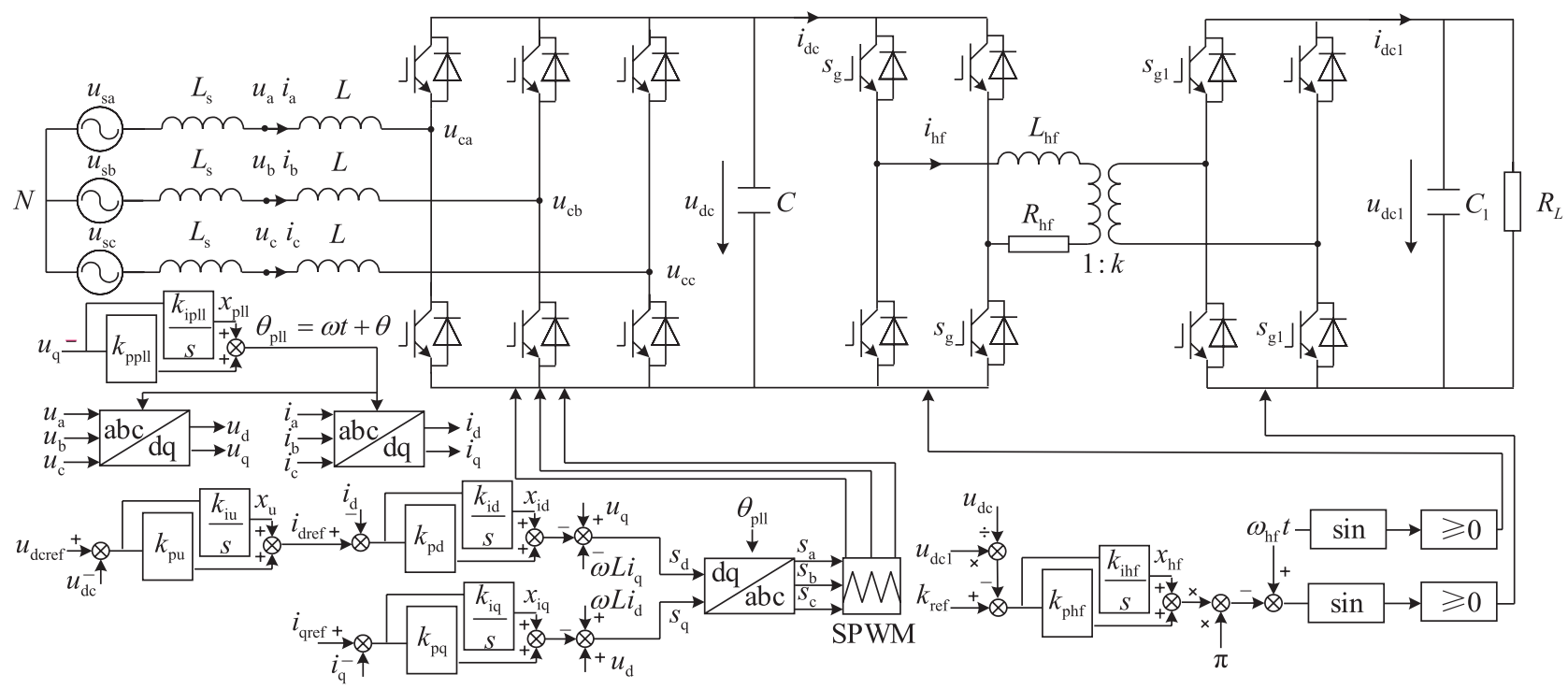

Fig. 3. The topology and control strategy of the two-stage high-frequency isolated AC-DC converter connected to AC grid.

$u_{\mathrm{dcref}}$ and $k_{\mathrm{ref}}$ are the instruction values for $i_{\mathrm{dq}}, u_{\mathrm{dc}}$ and the ratio of $u_{\mathrm{dcl}}$ and $u_{\mathrm{dc}} \cdot k_{\mathrm{pj}}$ and $k_{\mathrm{ij}}(\mathrm{j}=\mathrm{u}, \mathrm{d}, \mathrm{q}, \mathrm{hf}, \mathrm{pll})$ are the parameters of the PI controllers. $x_{\mathrm{j}}(\mathrm{j}=\mathrm{u}, \mathrm{d}, \mathrm{q}, \mathrm{hf}$, pll) are the integral outputs.

The conditions for two-stage high-frequency isolated AC$\mathrm{DC}$ converters to run stably at the instruction DC voltage are as follow:

(a) Power for maintaining DC voltage at instruction value can be transmitted by grid-connected AC-DC converters.

(b) The system is statically stable.

Therefore, there are two constraints to determine the range of VDI. One is the constraint on power transmission limit, the other is the constraint on static stability limit.

\section{A. Constraint on Power Transmission Limit}

The active power from the AC grid to the AC-DC converter is represented with $P_{\mathrm{c}}$. Usually unit power factor control is adopted by grid-connected AC-DC converters. According to Kirchhoff laws, (3) and (4) can be given as follows and their graphs are given in Fig. 4.

$$
\begin{aligned}
& U_{\mathrm{c}}^{2}=f_{\mathrm{c}}\left(U_{\mathrm{PCC}}^{2}\right)=U_{\mathrm{PCC}}^{2}+\left(\frac{P_{\mathrm{c}}}{3 U_{\mathrm{PCC}}} \omega L\right)^{2} \\
& U_{\mathrm{s}}^{2}=f_{\mathrm{s}}\left(U_{\mathrm{PCC}}^{2}\right)=U_{\mathrm{PCC}}^{2}+\left(\frac{P_{\mathrm{c}}}{3 U_{\mathrm{PCC}}} \omega L_{\mathrm{s}}\right)^{2}
\end{aligned}
$$

Where $U_{\mathrm{s}}, U_{\mathrm{PCC}}$ and $U_{\mathrm{c}}$ are steady-state root mean square of $u_{\text {sabc }}, u_{\text {abc }}$ and $u_{\text {cabc }}$.

Based on (3) and (4), (5) and (6) can be given as follows:

$$
\begin{aligned}
& U_{\mathrm{c}}^{2} \geqslant \frac{2 P_{\mathrm{c}} \omega L}{3} \\
& U_{\mathrm{s}}^{2} \geqslant \frac{2 P_{\mathrm{c}} \omega L_{\mathrm{s}}}{3}
\end{aligned}
$$

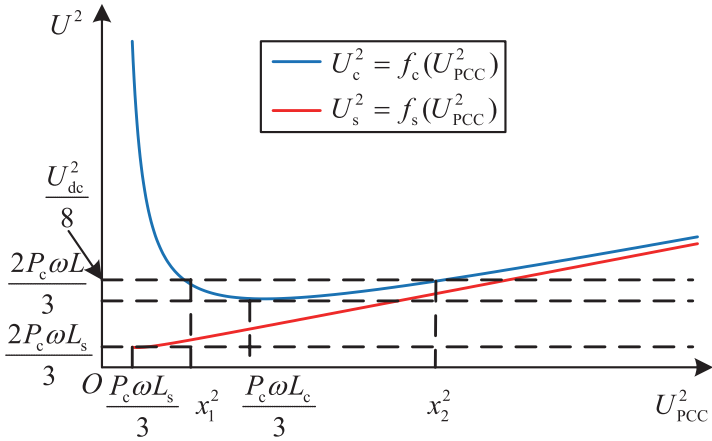

(a)

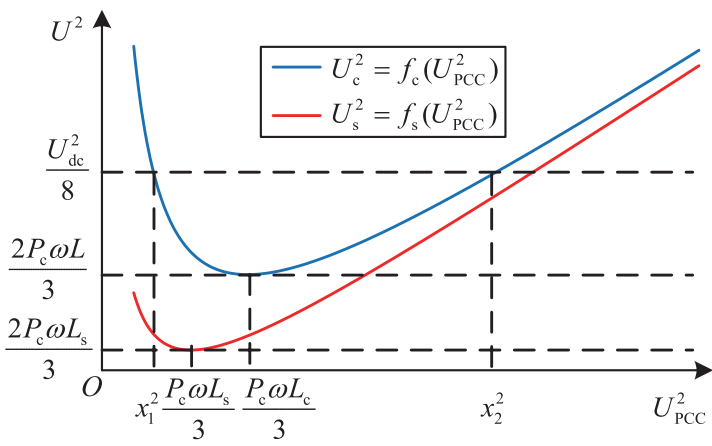

(b)

Fig. 4. Graphs of $U_{\mathrm{c}}^{2}=f_{\mathrm{c}}\left(U_{\mathrm{PCC}}^{2}\right)$ and $U_{\mathrm{s}}^{2}=f_{\mathrm{s}}\left(U_{\mathrm{PCC}}^{2}\right)$.

When $U_{\mathrm{PCC}}^{2}=\frac{P_{\mathrm{c}} \omega L}{3}$ or $\frac{P_{\mathrm{c}} \omega L_{\mathrm{s}}}{3}$, the equality in (5) or (6) holds. With the modulation ratio $M \leqslant 1$, (7) can be given as follows:

$$
U_{\mathrm{c}}^{2} \leqslant \frac{U_{\mathrm{dc}}^{2}}{8}
$$

Where $U_{\mathrm{dc}}$ is steady-state value of $u_{\mathrm{dc}}$. 
Substitute (3) into (7), (8) can be given as follows:

$$
x_{1} \leqslant U_{\mathrm{PCC}} \leqslant x_{2} \quad x_{1,2}=\sqrt{\frac{U_{\mathrm{dc}}^{2}}{16} \mp \sqrt{\frac{U_{\mathrm{dc}}^{4}}{256}-\frac{P_{\mathrm{c}}^{2} \omega^{2} L^{2}}{9}}}
$$

Substitute (8) into (4) and combine with (6).

When $x_{1}^{2} \geqslant \frac{P_{\mathrm{c}} \omega L_{\mathrm{s}}}{3}$, as shown in Fig. 4(a), $U_{\mathrm{smin}}=\sqrt{f_{\mathrm{s}}\left(x_{1}^{2}\right)}$,

$$
x_{1}^{2}<\frac{P_{\mathrm{c}} \omega L_{\mathrm{s}}}{3} \text {, as shown in Fig. 4(b), } U_{\mathrm{smin}}=\sqrt{\frac{2 P_{\mathrm{c}} \omega L_{\mathrm{s}}}{3}}
$$

The range of $U_{\mathrm{s}}$ with consideration on constraint on the power transmission limit is as follows:

$$
U_{\text {smin }} \leqslant U_{\mathrm{s}} \leqslant U_{\mathrm{s} \max }\left\{\begin{array}{l}
U_{\mathrm{s} \min }= \begin{cases}\sqrt{f_{\mathrm{s}}\left(x_{1}^{2}\right)} & x_{1}^{2} \geqslant \frac{P_{\mathrm{c}} \omega L_{\mathrm{s}}}{3} \\
\sqrt{\frac{2 P_{\mathrm{c}} \omega L_{\mathrm{s}}}{3}} & x_{1}^{2}<\frac{P_{\mathrm{c}} \omega L_{\mathrm{s}}}{3}\end{cases} \\
U_{\mathrm{s} \max }=\sqrt{f_{\mathrm{s}}\left(x_{2}^{2}\right)}
\end{array}\right.
$$

Based on (9), the range of VDI only with consideration on constraint on the power transmission limit can be given as follows:

$$
\begin{aligned}
& {\left[\Delta U_{\mathrm{v} 1}, \Delta U_{\mathrm{v} 2}\right]=\frac{\left[v_{1}, v_{2}\right]-U_{\mathrm{sn}}}{U_{\mathrm{sn}}} \times 100 \%} \\
& \begin{cases}v_{1}= \begin{cases}x_{1} & x_{1}^{2} \geqslant \frac{P_{\mathrm{c}} \omega L_{\mathrm{s}}}{3} \\
\sqrt{\frac{P_{\mathrm{c}} \omega L_{\mathrm{s}}}{3}} & x_{1}^{2}<\frac{P_{\mathrm{c}} \omega L_{\mathrm{s}}}{3}\end{cases} \\
v_{2}=x_{2}\end{cases}
\end{aligned}
$$

Where $U_{\mathrm{sn}}$ is the rated voltage of the AC grid.

When over modulation or space vector pulse width modulation (SVPWM) is considered, (7) is modified as follows:

$$
U_{\mathrm{c}}^{2} \leqslant \frac{2 U_{\mathrm{dc}}^{2}}{\pi^{2}}
$$

Substitute (3) into (11), (12) can be given as follows:

$$
x_{1} \leqslant U_{\mathrm{PCC}} \leqslant x_{2} \quad x_{1,2}=\sqrt{\frac{U_{\mathrm{dc}}^{2}}{\pi^{2}} \mp \sqrt{\frac{U_{\mathrm{dc}}^{4}}{\pi^{4}}-\frac{P_{\mathrm{c}}^{2} \omega^{2} L^{2}}{9}}}
$$

Similarly, the range of $U_{\mathrm{s}}$ and VDI with consideration on constraint on the power transmission limit can be obtained.

\section{B. Constraint on Static Stability Limit}

Based on Fig. 3, The equations of the main circuit of the front stage of the AC-DC converter are given as follows:

$$
\left\{\begin{array}{l}
\left(L+L_{\mathrm{s}}\right) \frac{d i_{\mathrm{j}}}{d t}=u_{\mathrm{sj}}-\frac{s_{\mathrm{j}} u_{\mathrm{dc}}}{2} \quad \mathrm{j}=\mathrm{a}, \mathrm{b}, \mathrm{c} \\
C \frac{d u_{\mathrm{dc}}}{d t}=\frac{1}{2} \sum_{\mathrm{j}=\mathrm{a}, \mathrm{b}, \mathrm{c}} s_{\mathrm{j}} i_{\mathrm{j}}-i_{\mathrm{dc}}
\end{array}\right.
$$

The transformation matrix is shown as (14).

$$
\begin{gathered}
{\left[\begin{array}{l}
\mathrm{d} \\
\mathrm{q}
\end{array}\right]=\frac{2}{3}\left[\begin{array}{ccc}
\cos \theta_{\mathrm{pll}} & \cos \left(\theta_{\mathrm{pll}}-\frac{2 \pi}{3}\right) & \cos \left(\theta_{\mathrm{pll}}+\frac{2 \pi}{3}\right) \\
\sin \theta_{\mathrm{pll}} & \sin \left(\theta_{\mathrm{pll}}-\frac{2 \pi}{3}\right) & \sin \left(\theta_{\mathrm{pll}}+\frac{2 \pi}{3}\right)
\end{array}\right]\left[\begin{array}{l}
\mathrm{a} \\
\mathrm{b} \\
\mathrm{c}
\end{array}\right](14)} \\
\theta_{\mathrm{pll}}=\omega t+\theta
\end{gathered}
$$

Transform (13) into dq coordinate.

$$
\left\{\begin{array}{l}
\left(L+L_{\mathrm{s}}\right) \frac{d i_{\mathrm{d}}}{d t}=u_{\mathrm{sd}}-\frac{s_{\mathrm{d}} u_{\mathrm{dc}}}{2}-\left(L+L_{\mathrm{s}}\right) \frac{d \theta_{\mathrm{pll}}}{d t} i_{\mathrm{q}} \\
\left(L+L_{\mathrm{s}}\right) \frac{d i_{\mathrm{q}}}{d t}=u_{\mathrm{sq}}-\frac{s_{\mathrm{q}} u_{\mathrm{dc}}}{2}+\left(L+L_{\mathrm{s}}\right) \frac{d \theta_{\mathrm{pll}}}{d t} i_{\mathrm{d}} \\
C \frac{d u_{\mathrm{dc}}}{d t}=\frac{3\left(s_{\mathrm{d}} i_{\mathrm{d}}+s_{\mathrm{q}} i_{\mathrm{q}}\right)}{4}-i_{\mathrm{dc}}
\end{array}\right.
$$

Where $u_{\text {sdq }}$ are the $u_{\text {sabc }}$ in dq coordinate.

The equations of the control circuit of the front stage are as follows:

$$
\begin{aligned}
& \left\{\begin{array}{l}
\frac{d x_{\mathrm{u}}}{d t}=u_{\mathrm{dcref}}-u_{\mathrm{dc}} \\
\frac{d x_{\mathrm{ij}}}{d t}=i_{\mathrm{jref}}-i_{\mathrm{j}} \quad \mathrm{j}=\mathrm{d}, \mathrm{q}
\end{array}\right. \\
& \left\{\begin{array}{l}
i_{\mathrm{dref}}=k_{\mathrm{pu}}\left(u_{\mathrm{dcref}}-u_{\mathrm{dc}}\right)+k_{\mathrm{iu}} x_{\mathrm{u}} \\
i_{\mathrm{qref}}=0 \\
s_{\mathrm{d}}=u_{\mathrm{d}}-\omega L i_{\mathrm{q}}-k_{\mathrm{pd}}\left(i_{\mathrm{dref}}-i_{\mathrm{d}}\right)-k_{\mathrm{id}} x_{\mathrm{id}} \\
s_{\mathrm{q}}=u_{\mathrm{q}}+\omega L i_{\mathrm{d}}-k_{\mathrm{pq}}\left(i_{\mathrm{qref}}-i_{\mathrm{q}}\right)-k_{\mathrm{iq}} x_{\mathrm{iq}}
\end{array}\right.
\end{aligned}
$$

The equation of phase-locked loop (PLL) is as follows:

$$
\left\{\begin{array}{l}
\frac{d \theta}{d t}=-\omega-k_{\mathrm{ppll}} u_{\mathrm{q}}-k_{\mathrm{ipl1}} x_{\mathrm{pll}} \\
\frac{d x_{\mathrm{pll}}}{d t}=u_{\mathrm{q}}
\end{array}\right.
$$

Based on Fig. 3, the equations of the main circuit of the back stage of the AC-DC converter are given as follows:

$$
\left\{\begin{array}{c}
L_{\mathrm{hf}} \frac{d i_{\mathrm{hf}}}{d t}=s_{\mathrm{g}} u_{\mathrm{dc}}-\frac{s_{\mathrm{g} 1} u_{\mathrm{dc} 1}}{k}-R_{\mathrm{hf}} i_{\mathrm{hf}} \\
C_{1} \frac{d u_{\mathrm{dc} 1}}{d t}=\frac{s_{\mathrm{g} 1} i_{\mathrm{hf}}}{k}-\frac{u_{\mathrm{dc} 1}}{R_{L}} \\
i_{\mathrm{hf}}=s_{\mathrm{g}} i_{\mathrm{dc}}
\end{array}\right.
$$

The transformation matrix is shown as (22).

$$
\left[\begin{array}{l}
\mathrm{d} \\
\mathrm{q}
\end{array}\right]=\frac{2}{3}\left[\begin{array}{ccc}
\cos \omega_{\mathrm{hf}} t & \cos \left(\omega_{\mathrm{hf}} t-\frac{2 \pi}{3}\right) & \cos \left(\omega_{\mathrm{hf}} t+\frac{2 \pi}{3}\right) \\
\sin \omega_{\mathrm{hf}} t & \sin \left(\omega_{\mathrm{hf}} t-\frac{2 \pi}{3}\right) & \sin \left(\omega_{\mathrm{hf}} t+\frac{2 \pi}{3}\right)
\end{array}\right]\left[\begin{array}{l}
\mathrm{a} \\
\mathrm{b} \\
\mathrm{c}
\end{array}\right]
$$


Extend (20) to the equations in three phases and transform them into dq coordinate.

$$
\left\{\begin{array}{l}
L_{\mathrm{hf}} \frac{d i_{\mathrm{hfd}}}{d t}=s_{\mathrm{gd}} u_{\mathrm{dc}}-\frac{s_{\mathrm{gld}} u_{\mathrm{dc} 1}}{k}-\omega_{\mathrm{hf}} L_{\mathrm{hf}} i_{\mathrm{hfq}}-R_{\mathrm{hf}} i_{\mathrm{hfd}} \\
L_{\mathrm{hf}} \frac{d i_{\mathrm{hfq}}}{d t}=s_{\mathrm{gq}} u_{\mathrm{dc}}-\frac{s_{\mathrm{g} 1 \mathrm{q}} u_{\mathrm{dc} 1}}{k}+\omega_{\mathrm{hf}} L_{\mathrm{hf}} i_{\mathrm{hfd}}-R_{\mathrm{hf}} i_{\mathrm{hfq}} \\
C_{1} \frac{d u_{\mathrm{dc} 1}}{d t}=\frac{s_{\mathrm{g} 1 \mathrm{~d}} i_{\mathrm{hfd}}+s_{\mathrm{g} 1 \mathrm{q}} i_{\mathrm{hfq}}}{2 k}-\frac{u_{\mathrm{dc} 1}}{R_{L}}
\end{array}\right.
$$

Where $s_{\mathrm{gdq}}, s_{\mathrm{gldq}}$ and $i_{\mathrm{hfdq}}$ are $s_{\mathrm{g}}, s_{\mathrm{g} 1}$ and $i_{\mathrm{hf}}$ in dq coordinate.

The equations of the control circuit of the back stage are as follows:

$$
\begin{gathered}
\frac{d x_{\mathrm{hf}}}{d t}=k_{\mathrm{ref}}-\frac{u_{\mathrm{dc} 1}}{u_{\mathrm{dc}}} \\
\left\{\begin{array}{l}
s_{\mathrm{g}}=\frac{4}{\pi} \sin \omega_{\mathrm{hf}} t \\
s_{\mathrm{g} 1}=\frac{4}{\pi} \sin \left\{\omega_{\mathrm{hf}} t-\left[k_{\mathrm{phf}}\left(k_{\mathrm{ref}}-\frac{u_{\mathrm{dc} 1}}{u_{\mathrm{dc}}}\right)+k_{\mathrm{ihf}} x_{\mathrm{hf}}\right] \pi\right\}
\end{array}\right.
\end{gathered}
$$

Transform (25) into dq coordinate.

$$
\left\{\begin{array}{l}
s_{\mathrm{gd}}=0 \quad s_{\mathrm{gq}}=\frac{4}{\pi} \\
s_{\mathrm{gld}}=-\frac{4}{\pi} \sin \left[k_{\mathrm{phf}}\left(k_{\mathrm{ref}}-\frac{u_{\mathrm{dcl}}}{u_{\mathrm{dc}}}\right)+k_{\mathrm{ihf}} x_{\mathrm{hf}}\right] \pi \\
s_{\mathrm{glq}}=\frac{4}{\pi} \cos \left[k_{\mathrm{phf}}\left(k_{\mathrm{ref}}-\frac{u_{\mathrm{dcl}}}{u_{\mathrm{dc}}}\right)+k_{\mathrm{ihf}} x_{\mathrm{hf}}\right] \pi
\end{array}\right.
$$

Based on the above equations, select $\boldsymbol{X}$ as state variables and the state-space average model of the two-stage high-frequency isolated AC-DC converter can be obtained.

$$
\boldsymbol{X}=\left[i_{\mathrm{d}}, i_{\mathrm{q}}, u_{\mathrm{dc}}, x_{\mathrm{u}}, x_{\text {id }}, x_{\mathrm{iq}}, \theta, x_{\mathrm{pll}}, i_{\mathrm{hfd}}, i_{\mathrm{hfq}}, u_{\mathrm{dc} 1}, x_{\mathrm{hf}}\right]^{\mathrm{T}}
$$

Set the derivative of the state variable to 0 and obtain (28) and (29) by ignoring the power of $R_{\mathrm{hf}}$. The steady-state point of the model can be approximately obtained by analytic expressions rather than simulation and numerical calculation.

$$
\begin{aligned}
& \tilde{u_{\mathrm{sd}}}=\sqrt{\frac{U_{\mathrm{s}}^{2}}{2}+\frac{\omega L_{\mathrm{s}} k_{\mathrm{ref}}^{2} u_{\mathrm{dcref}}^{2}}{3 R_{L}}}+\sqrt{\frac{U_{\mathrm{s}}^{2}}{2}-\frac{\omega L_{\mathrm{s}} k_{\mathrm{ref}}^{2} u_{\mathrm{dcref}}^{2}}{3 R_{L}}} \\
& u_{\mathrm{sq}}^{\sim}= \begin{cases}\sqrt{2 U_{\mathrm{s}}^{2}-u_{\mathrm{sd}}^{\sim 2}} & P_{\mathrm{c}}<0 \\
-\sqrt{2 U_{\mathrm{s}}^{2}-u_{\mathrm{sd}}^{\sim 2}} & P_{\mathrm{c}} \geqslant 0\end{cases} \\
& \left\{\tilde{i_{\mathrm{d}}}=\frac{2 k_{\mathrm{ref}}^{2} u_{\mathrm{dcref}}^{2}}{3 R_{L} \tilde{u_{\mathrm{sd}}}} \quad i_{\mathrm{q}}^{\sim}=i_{\mathrm{qref}} \quad \tilde{u_{\mathrm{dc}}}=u_{\mathrm{dcref}} \quad \tilde{x_{\mathrm{u}}}=\frac{\tilde{i_{\mathrm{d}}}}{k_{\mathrm{iu}}}\right. \\
& \tilde{x_{\mathrm{id}}}=\frac{\left(\tilde{u_{\mathrm{dc}}}-2\right) \tilde{u_{\mathrm{sd}}}}{u_{\mathrm{dc}}^{\sim} k_{\mathrm{id}}} \quad \tilde{x_{\mathrm{iq}}}=\frac{2 L u_{\mathrm{sq}}^{\sim}+\omega L L_{\mathrm{s}} \tilde{u_{\mathrm{dc}}} \tilde{i_{\mathrm{d}}}}{L_{\mathrm{s}} u_{\mathrm{dc}}^{\sim} k_{\mathrm{iq}}} \\
& \theta^{\sim}=-\arctan \left(\frac{\tilde{u_{\mathrm{sq}}}}{\tilde{u_{\mathrm{sd}}}}\right) \quad \tilde{x_{\mathrm{pll}}}=\frac{\omega}{k_{\mathrm{ipll}}}
\end{aligned}
$$

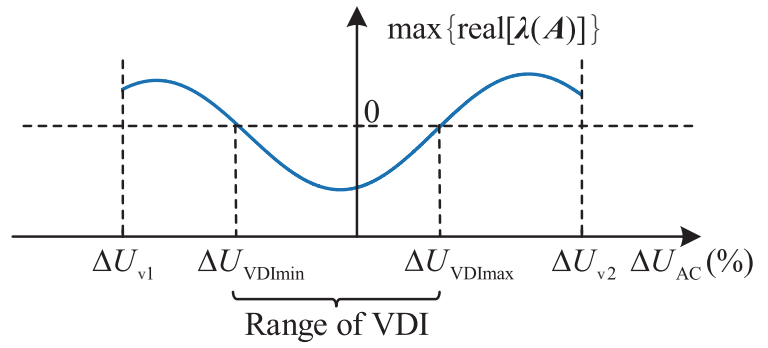

Fig. 5. Determination of the range of VDI with both constraints on power transmission limit and static stability limit.

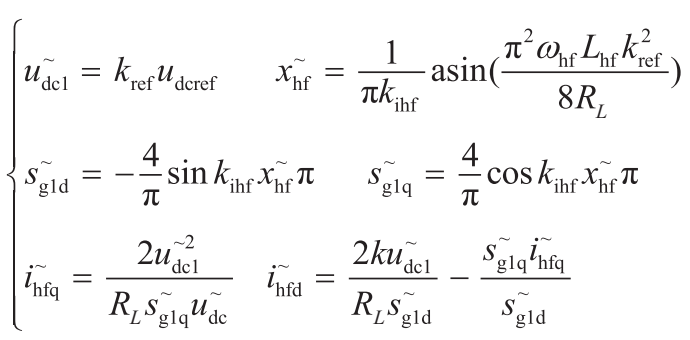

Where $\langle\cdot\rangle^{\sim}$ represent the steady-state value of $\langle\cdot\rangle$.

Through linearizing the model at the steady-state point, the small signal model is obtained [17], as follows:

$$
\frac{d \Delta \boldsymbol{X}}{d t}=\boldsymbol{A} \Delta \boldsymbol{X}+\boldsymbol{B} \Delta \boldsymbol{Y}
$$

The constraint on the static stability limit of high-frequency isolated AC-DC converter is as follow:

$$
\max \{\operatorname{real}[\lambda(\boldsymbol{A})]\}<0
$$

Where real $[\lambda(\boldsymbol{A})]$ represents the real parts of the eigenvalues of matrix $\boldsymbol{A}$.

\section{Determination of Range of VDI}

The range of VDI can be determined as shown in Fig. 5. Based on (10), the range of abscissa is $\left[\Delta U_{\mathrm{v} 1}, \Delta U_{\mathrm{v} 2}\right]$. According to (31), $\left[\Delta U_{\text {VDImin }}, \Delta U_{\text {VDImax }}\right]$ can be determined.

The flow chart of the proposed method for determination of the range of VDI for two-stage high-frequency isolated AC-DC converters with constant DC voltage control was given in Fig. 6. Actually, the method is available for other types of AC-DC converters with constant DC voltage control in LVDC systems.

\section{Simulation Test}

\section{A. Parameters of Simulation Model}

A simulation model of the two-stage high-frequency isolated AC-DC converter is established based on Fig. 3. The parameters of the simulation model are shown in Table I.

\section{B. Calculation Results}

According to the proposed method, the ranges of $U_{\mathrm{s}}$ and 


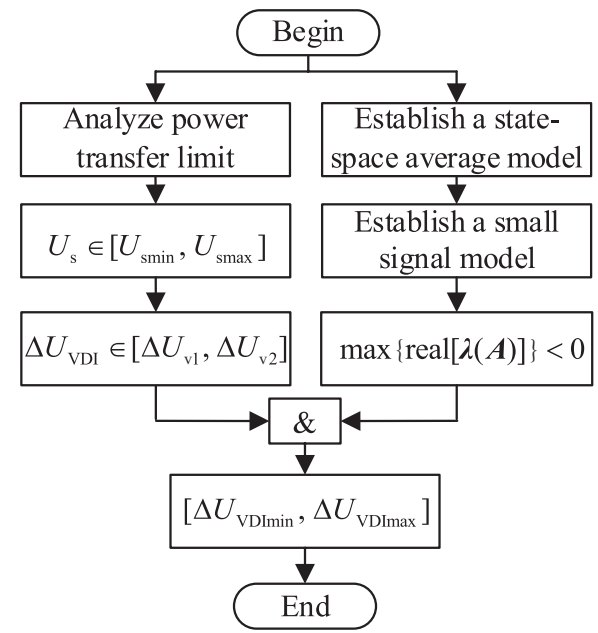

Fig. 6. Method for determination of the range of VDI for two-stage highfrequency isolated AC-DC converters with constant DC voltage control.

TABLE I

Parameters of the Simulation Model

\begin{tabular}{lccc}
\hline \hline Parameter & Value & Parameter & Value \\
\hline$U_{\text {sn }}$ & $220 \mathrm{~V}$ & $u_{\text {dcref }}$ & $700 \mathrm{~V}$ \\
$\omega$ & $100 \pi \mathrm{rad} / \mathrm{s}$ & $k_{\mathrm{pu}}$ & 10 \\
$L_{\mathrm{s}}$ & $30 \mu \mathrm{H}$ & $k_{\text {iu }}$ & 1000 \\
$L$ & $600 \mu \mathrm{H}$ & $i_{\mathrm{qref}}$ & $0 \mathrm{~A}$ \\
$C$ & $6300 \mu \mathrm{F}$ & $k_{\mathrm{pd} / \mathrm{q}}$ & 5 \\
$C_{1}$ & $3300 \mu \mathrm{F}$ & $k_{\text {id/q }}$ & 100 \\
$\omega_{\mathrm{hf}}$ & $2 \pi \times 10^{4} \mathrm{rad} / \mathrm{s}$ & $k_{\mathrm{ppll}}$ & 578 \\
$R_{\mathrm{hf}}$ & $0.001 \Omega$ & $k_{\text {ipll }}$ & 10285 \\
$L_{\mathrm{hf}}$ & $25 \mu \mathrm{H}$ & $k_{\text {ref }}$ & $0.75 / 0.7$ \\
$k$ & $0.75 / 0.7$ & $k_{\mathrm{phf}}$ & 0.01 \\
$R_{L}$ & $5.625 \Omega$ & $k_{\text {ihf }}$ & 10 \\
\hline \hline
\end{tabular}

TABLE II

Ranges of $U_{\mathrm{s}}$ AND VDI OnLy With Consideration on Constraint on Power TRANSMission Limit

\begin{tabular}{lcccc}
\hline \hline Modulation Ratio & $U_{\text {smin }}$ & $U_{\text {smax }}$ & $\Delta U_{\mathrm{v} 1}$ & $\Delta U_{\mathrm{v} 2}$ \\
\hline $0<M \leqslant 1$ & $28.3 \mathrm{~V}$ & $246.2 \mathrm{~V}$ & $-88.4 \%$ & $+11.9 \%$ \\
$0<M \leqslant \frac{4}{\pi}$ & $25.4 \mathrm{~V}$ & $314.5 \mathrm{~V}$ & $-90.9 \%$ & $+42.9 \%$ \\
\hline \hline
\end{tabular}

VDI only with consideration on constraint on the power transmission limit are given in Table II. The range of VDI is shown in Fig. 7. If over modulation or SVPWM is considered, the range of VDI is $[-51.9 \%,+42.9 \%]$, else the range of VDI is $[-51.9 \%,+11.9 \%]$.

\section{Simulation Results}

To testify the correctness of the calculation results, three scenarios are selected as shown in Table III.

In scenario $1,-88.4 \%<\Delta U_{\mathrm{AC}}=-60 \%<-51.9 \%$, which satisfies the constraint on the power transmission limit but not the static stability limit. The simulation results are shown in Fig. 8. $u_{\mathrm{dcl}}$ oscillates around instruction voltage when $i_{\mathrm{abc}}$ and $s_{\text {abc }}$ also oscillate, which means the converter can transmit the

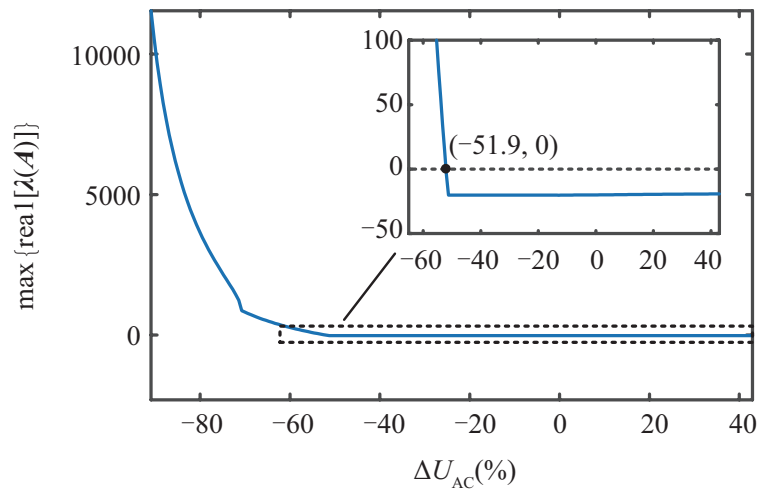

Fig. 7. Range of VDI for the two-stage high-frequency isolated AC-DC converter.

TABLE III

Three Scenarios to Simulate

\begin{tabular}{lccc}
\hline \hline Number & 1 & 2 & 3 \\
\hline$U_{\mathrm{s}}$ & $88.1 \mathrm{~V}$ & $132.0 \mathrm{~V}$ & $264.0 \mathrm{~V}$ \\
$\Delta U_{\mathrm{AC}}$ & $-60 \%$ & $-40 \%$ & $+20 \%$ \\
\hline \hline
\end{tabular}
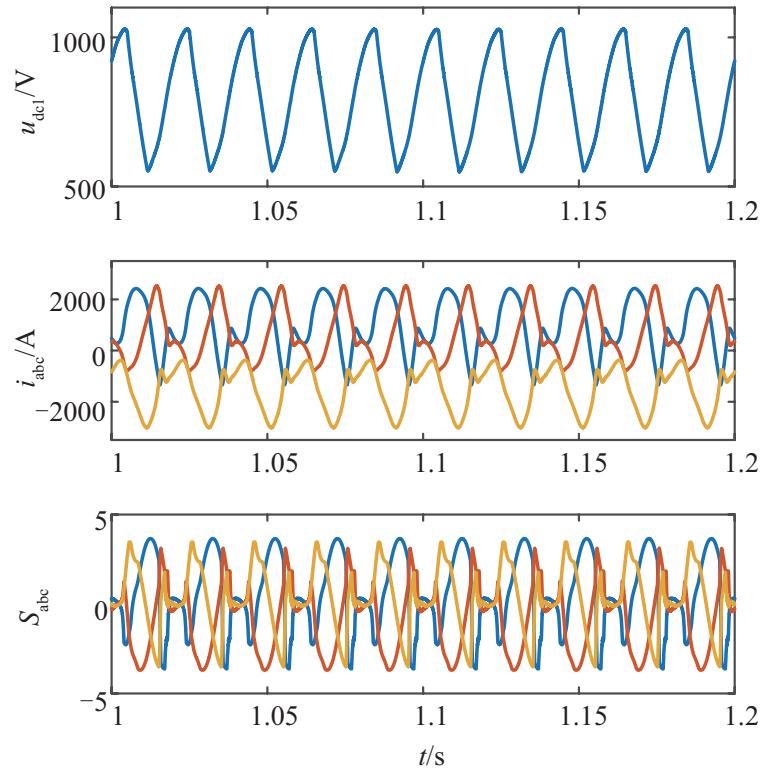

Fig. 8. Waveforms of the currents at the AC side, voltage at the DC side and modulation signals in scenario 1 .

power in need but not keep stable.

In scenario $2,-51.9 \%<\Delta U_{\mathrm{AC}}=-40 \%<+11.9 \%$, which satisfies the constraint on the power transmission limit and the static stability limit with $M \leqslant 1$. The simulation results are shown in Fig. 9. $s_{\mathrm{abc}}$ are between -1 and 1. $u_{\mathrm{dcl}}$ is stabilized at the instruction voltage which means the converter can stably transmitted the power in need without over modulation.

In scenario $3,+11.9 \%<\Delta U_{\mathrm{AC}}=+20 \%<+42.9 \%$, which satisfies the constraint on the power transmission limit and the static stability limit with $M>1$. The simulation results are shown in Fig. 10. $u_{\mathrm{dcl}}$ is stabilized at the instruction voltage and 

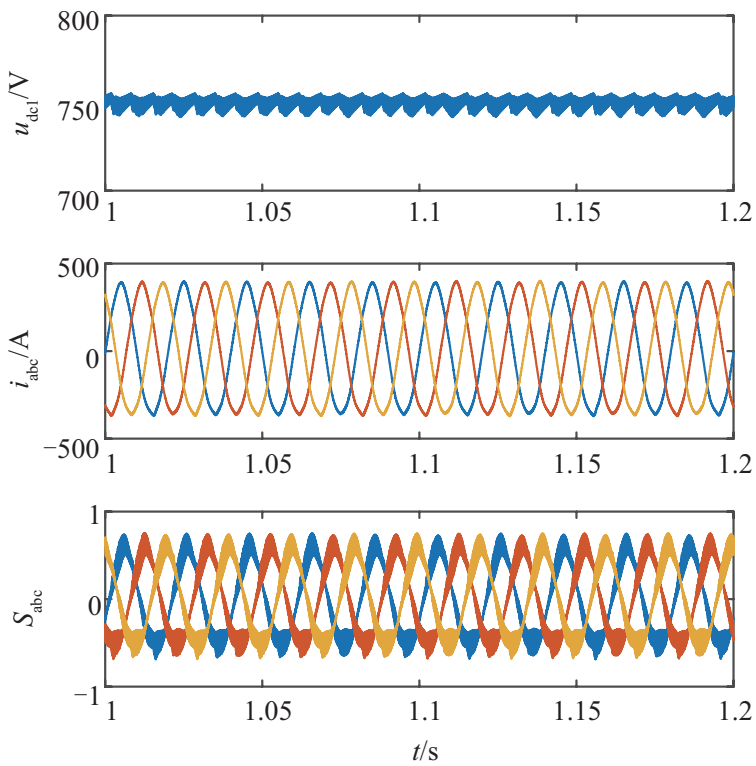

Fig. 9. Waveforms of the currents at the $\mathrm{AC}$ side, voltage at the $\mathrm{DC}$ side and modulation signals in scenario 2 .
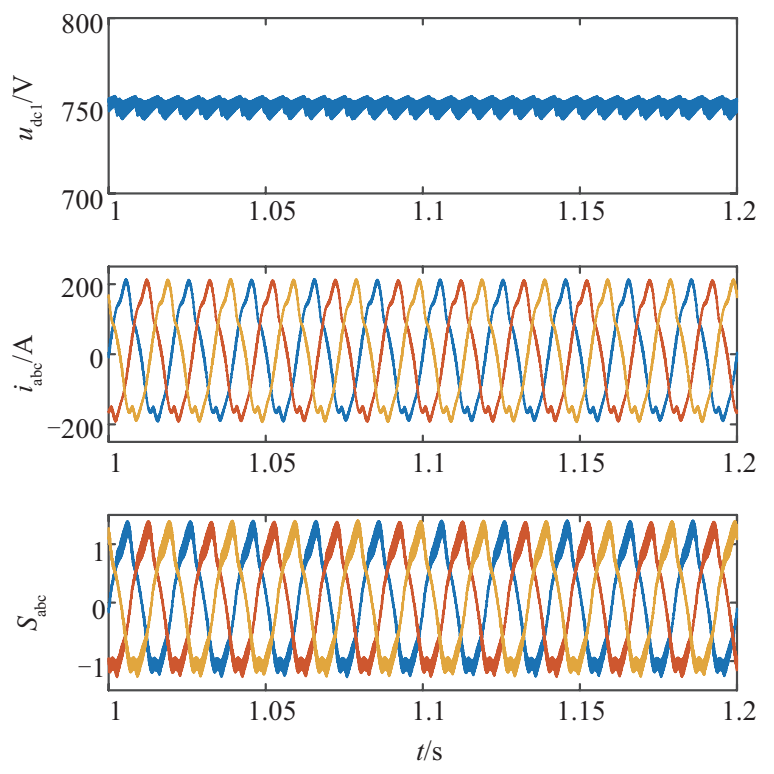

Fig. 10. Waveforms of the currents at the AC side, voltage at the DC side and modulation signals in scenario 3 .

$S_{\mathrm{abc}}$ are out of the range from -1 to 1 . Due to over modulation, $i_{\text {abc }}$ have more harmonics in scenario 3 than in scenario 2 .

The simulation results in three scenarios are in accord with the theoretical analysis so that the feasibility and validity of the proposed method are verified.

\section{Analysis of Influence Factors of Range of VDI}

Several factors are selected to research their influences on the range of VDI, including parameters of PI controllers, the impedance of AC grid, transmitted power and DC output voltage. The factors probably affecting the range of VDI are analyzed based on the AC-DC converter given in section IV.

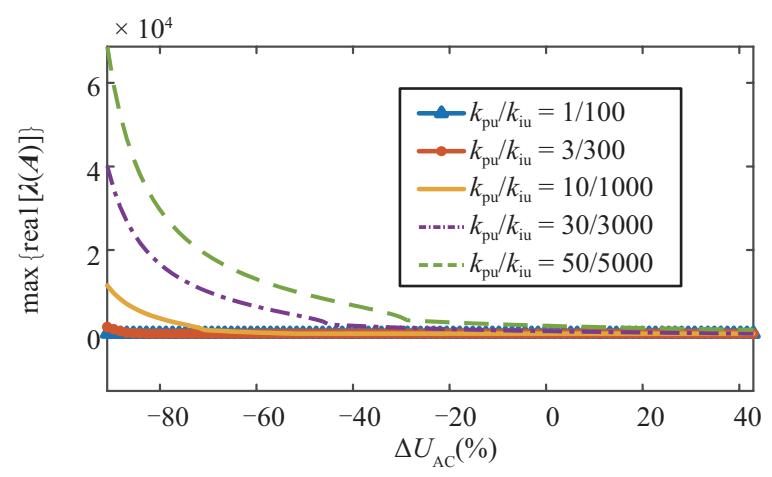

Fig. 11. Ranges of VDI with different $k_{\mathrm{pu}} / k_{\mathrm{uu}}$.

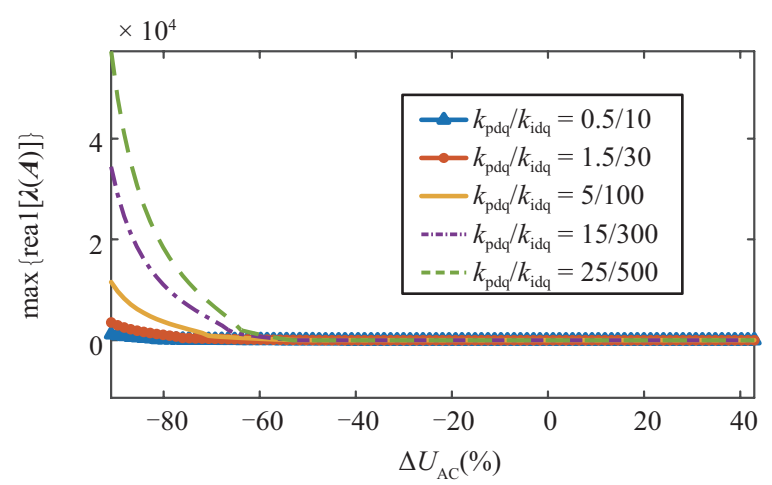

Fig. 12. Ranges of VDI with different $k_{\text {pdq }} / k_{\text {idq. }}$

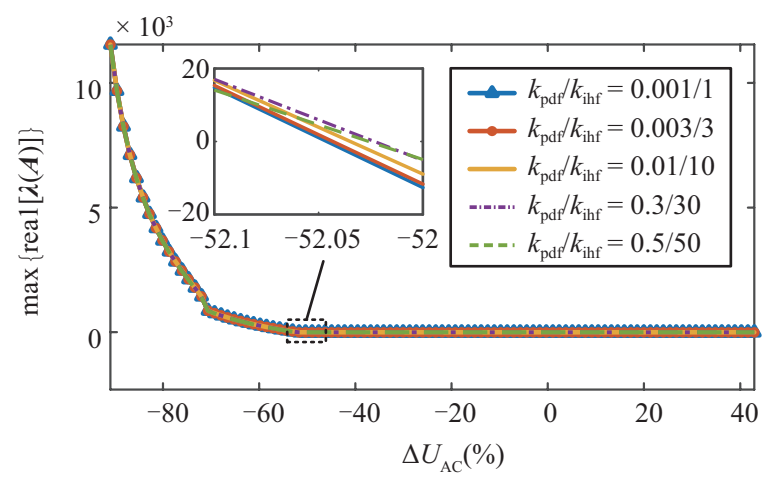

Fig. 13. Ranges of VDI with different $k_{\mathrm{pht}} / k_{\mathrm{ihf}}$.

\section{A. Ranges of VDI With Different Parameters of PI Controllers}

Ranges of VDI with different $k_{\mathrm{pu}} / k_{\mathrm{iu}}, k_{\mathrm{pdq}} / k_{\mathrm{idq}}$ and $k_{\mathrm{pht}} / k_{\mathrm{inf}}$ are shown in Fig. 11, Fig. 12 and Fig. 13 respectively.

In Fig. 11 and Fig. 12, as the parameters of PI controllers increase, the range of VDI $\left[\Delta U_{\mathrm{vl}}, \Delta U_{\mathrm{v} 2}\right]$ with only consideration on power transmitted limit remains the same, $\Delta U_{\text {VDImin }}$ increases and the range of VDI $\left[\Delta U_{\text {VDIminin }}, \Delta U_{\text {VDImax }}\right]$ shrinks. It is because the parameters of PI controllers only affect the static stability limit but not the power transmitted limit and the larger the parameters are, the worse the static stability is. In Fig. 13, the phenomenon is similar but not obvious, which means the range of VDI of the high-frequency isolated AC-DC converters is 


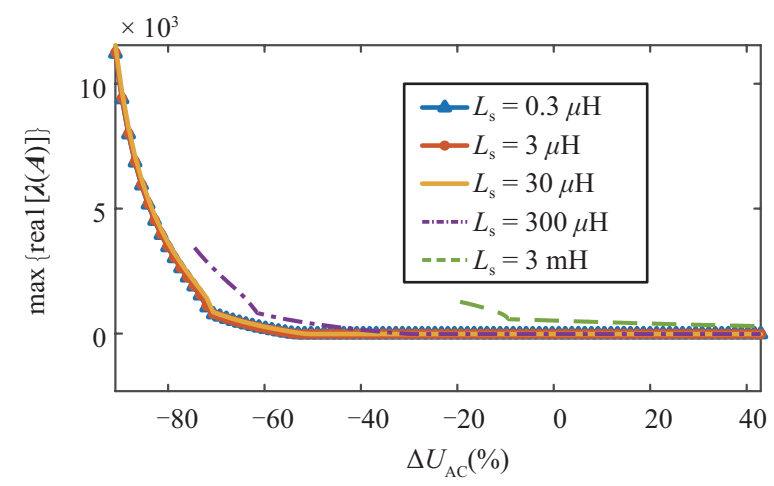

Fig. 14. Ranges of VDI with different $L_{\mathrm{s}}$.

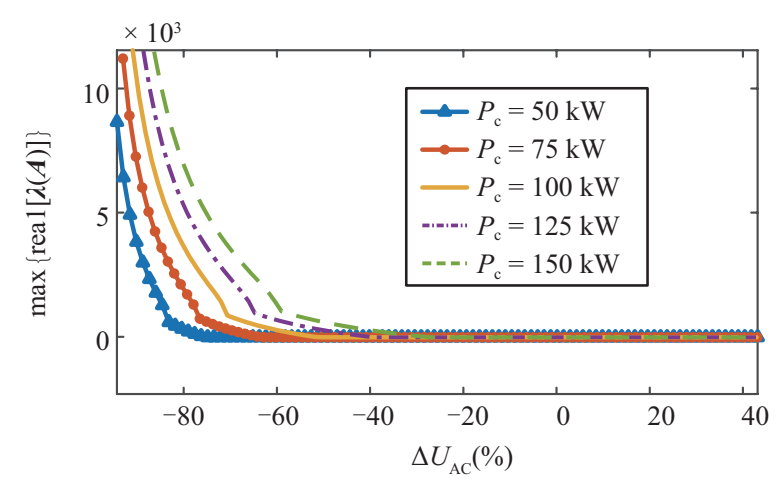

Fig. 15. Ranges of VDI with different $P_{\mathrm{c}}$.

more sensitive to $k_{\mathrm{pu}} / k_{\mathrm{iu}}, k_{\mathrm{pdq}} / k_{\mathrm{idq}}$ than $k_{\mathrm{phf}} / k_{\mathrm{ihf}}$.

\section{B. Ranges of VDI With Different Equivalent Impedances of AC GRID}

Ranges of VDI with different equivalent impedances of the AC grid are shown in Fig. 14. As $L_{\mathrm{s}}$ increase, $\Delta U_{\mathrm{v} 1}$ increases, $\Delta U_{\text {VDImin }}$ increases and the range of VDI shrinks. It is because the increase of $L_{\mathrm{s}}$, which reduce the strength of the AC grid, can reduce the power transmission limit and deteriorates the static stability.

\section{Ranges of VDI With Different Transmitted Power}

Ranges of VDI with different transmitted power of the AC grid are shown in Fig. 15. As $P_{\mathrm{c}}$ increases, $\Delta U_{\mathrm{v} 1}$ increases and the range of VDI shrinks because of the increase of $\Delta U_{\text {VDImin }}$ caused by the worse static stability.

\section{Ranges of VDI With Different DC Output Voltages}

According to Fig. $3, \tilde{u_{\mathrm{dc}}}{ }$ is changed by setting different $k_{\text {ref }}$ and the transmitted power $P_{\mathrm{c}}$ remains the same by adjusting $R_{\mathrm{L}}$. Range of VDI with different $k_{\text {ref }}$ is shown in Fig. 16.

As the $k_{\text {ref }}$ increases, $\tilde{u_{\mathrm{dcl}}}$ increases but range of VDI barely changes. The reason is that it is $u_{\mathrm{dc}}$ directly involved in the modulation of $u_{\text {cabc }}$ but not $u_{\mathrm{dc} 1}$. Because $P_{\mathrm{c}}$ and $\tilde{u_{\mathrm{dc}}}$ remain the same, the front stage of the AC-DC converter stays at the same

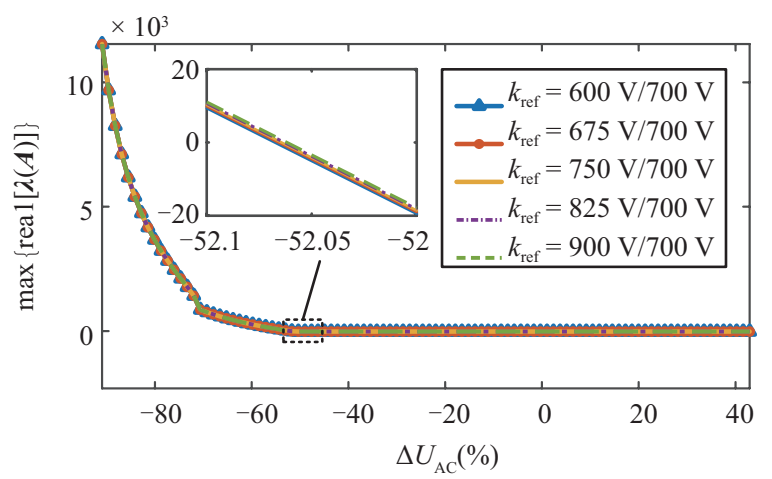

Fig. 16. Ranges of VDI with different $k_{\text {ref. }}$

condition. The $k_{\text {ref }}$ varies by only $\pm 20 \%$ so that the back stage can maintain normal operation. Therefore, the range of VDI is barely affected by DC output voltage.

\section{CONCLUSION}

In this paper, VDIR and the range of VDI are defined to assess the VDI performance and applied in the two-stage highfrequency AC-DC converters with constant DC voltage control in LVDC systems. A method is proposed to analyze the range of VDI based on the power transmission limit and the static stability limit. The main conclusions are as follows:

(a) The method proposed is useful to determine the range of VDI for two-stage high-frequency AC-DC converters with constant DC voltage control in LVDC systems. Actually, the method is available for various types of grid-connected ACDC converters in LVDC systems.

(b) The findings from the analysis of the influence factors of the range of VDI are shown as follows.

For two-stage high-frequency AC-DC converters in LVDC systems, the lower and upper bounds of the range of VDI are determined by constraints on static stability limit and power transmission limit.

As parameters of $\mathrm{PI}$ controllers, the impedance of $\mathrm{AC}$ grid or transmitted power increase, the range of VDI shrinks with the increase of the lower bound. And the range of VDI is barely affected by DC output voltage when the transmitted power remains the same.

In the next research stage, other control strategies will be considered in the analysis of VDI, which can bring more interesting findings. And the isolation ratio proposed in the paper is not only suitable for voltage deviation, but also can be applied for harmonic, voltage dip and other power quality problems, which may be the future research direction.

\section{REFERENCES}

[1] T. Dragicevic, J. C. Vasquez, J. M. Guerrero, and D. Skrlec, "Advanced LVDC electrical power architectures and microgrids: A step toward a new generation of power distribution networks," in IEEE Electrification Magazine, vol. 2, no. 1, pp. 54-65, Mar. 2014.

[2] G. Gwon, D. Kim, Y. Oh, J. Han, and C. Kim, "Analysis of efficiency for AC and DC load in LVDC distribution system," in Proceedings 
of 12th IET International Conference on Developments in Power System Protection (DPSP 2014), Copenhagen, Denmark, Mar. 2014, pp. 1-5.

[3] Y. Cho, J. Kim, J. Cho, and J. Kim, "Design and construction of LVDC distribution site," in Proceedings of CIRED Workshop 2016, Helsinki, Jun. 2016, pp. 1-4.

[4] J. Barros, M. De Apráiz, and R. I. Diego, "Definition and measurement of power quality indices in low voltage DC networks," in Proceedings of 2018 IEEE 9th International Workshop on Applied Measurements for Power Systems (AMPS), Bologna, Italy, Sept. 2018, pp. 1-5.

[5] H. Kakigano, Y. Miura, T. Ise, and R. Uchida, "DC voltage control of the DC micro-grid for super high quality distribution," in Proceedings of 2007 Power Conversion Conference-Nagoya, Nagoya, Japan, Apr. 2007, pp. $518-525$

[6] P. Mahirane, K. Park, J. Cha, J. Go, T. Choi, J. Oh, and J. Kim, "Analysis of voltage variation in LVDC distribution system with load variability," in Proceedings of 2017 20th International Conference on Electrical Machines and Systems (ICEMS), Sydney, NSW Australia, Aug. 2017, pp. 1-4.

[7] T. -H. Jung, G. -H. Gwon, C. -H. Kim, J. Han, Y. -S. Oh, and C. -H. Noh, "Voltage regulation method for voltage drop compensation and unbalance reduction in bipolar low-voltage DC distribution system," in IEEE Transactions on Power Delivery, vol. 33, no. 1, pp. 141-149, Feb. 2018.

[8] B. Morris, G. Faheem, R. Enrico, and Z. Dario, "Modeling of LVDC distribution system: An assessment of control, power quality, and DC faults," in Proceedings of 2020 19th International Conference on Harmonics and Quality of Power (ICHQP), Dubai, United Arab Emirates, Jul. 2020, pp. 1-5.

[9] K. Sumiya, K. Sakuma, J. Xu, N. Shimosato, and Y. Sato, "Suppression control of lower-order harmonics of input current in bidirectional isolated three-phase $\mathrm{AC} / \mathrm{DC}$ dual-active-bridge converter based on matrix converter," in Proceedings of 2019 10th International Conference on Power Electronics and ECCE Asia (ICPE 2019-ECCE Asia), Busan, Korea (South), May 2019, pp. 1-6.

[10] O. Babayomi, Z. Li, and Z. Zhang, "Distributed secondary frequency and voltage control of parallel-connected vscs in microgrids: A predictive VSG-based solution," in CPSS Transactions on Power Electronics and Applications, vol. 5, no. 4, pp. 342-351, Dec. 2020.

[11] T. Gonen, in Electrical Machines With MATLAB, 2nd ed. CRC Press, USA, 2011.

[12] Z. Linn, K. Shigeuchi, and Y. Sato, "Performance analysis of high frequency isolated AC/DC converter based on matrix converter," in Proceedings of 2018 15th International Conference on Electrical Engineering/Electronics, Computer, Telecommunications and Information Technology (ECTI-CON), Chiang Rai, Thailand, Jul. 2018 , pp. 286-289.

[13] J. E. Huber, J. Böhler, D. Rothmund, and J. W. Kolar, "Analysis and celllevel experimental verification of a $25 \mathrm{~kW}$ all-SiC isolated front end 6.6 $\mathrm{kV} / 400 \mathrm{~V}$ AC-DC solid-state transformer," in CPSS Transactions on Power Electronics and Applications, vol. 2, no. 2, pp. 140-148, Jun. 2017.

[14] S. -H. Lee and M. -J. Kim, "High efficiency isolated resonant PFC converter for two-stage AC-DC converter with enhanced performance," in Proceedings of 2019 IEEE Energy Conversion Congress and Exposition (ECCE), Baltimore, MD, USA, Sept. 2019, pp. 1120-1124.

[15] K. Sakuma, K. Shigeuchi, J. Xu, N. Shimosato, and Y. Sato, "Decoupling control method for eliminating DC bias flux of high frequency transformer in a bidirectional isolated AC/DC converter," in Proceedings of 2018 International Power Electronics Conference (IPEC-Niigata 2018 -ECCE Asia), Niigata, Japan, May 2018, pp. 3522-3527.

[16] N. Hirose, Y. Matsui, and T. Takeshita, "Isolated AC/DC converter using simple PWM strategy," in Proceedings of 2018 International Power Electronics Conference (IPEC-Niigata 2018 -ECCE Asia), Niigata, Japan, May 2018, pp. 3791-3796.

[17] P. M. Anderson and A. A. Fouad, "System response to small disturbances," in Power System Control and Stability, IEEE, 2003, pp. 53-80.

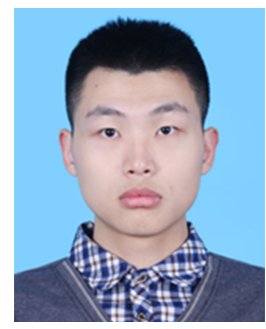

Yangxin Qiu received the B.Sc. degree in South China University of Technology, Guangzhou, China, in 2017. He is now a PhD student in School of Electric Power, South China University of Technology. His main fields of interest include power quality and power electronics control.

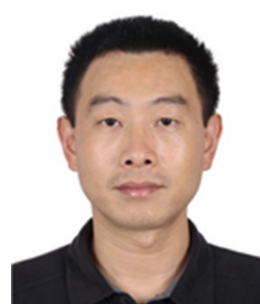

Qing Zhong received Ph.D. and M.Sc. degrees in South China University of Technology, Guangzhou, China in 2003 and 2000, and B.Sc. degree in North China University of Technology, Beijing, China, in 1997, all in electrical engineering. He is now a Professor in School of Electric Power, South China University of Technology. His main research interests include power quality and power electronics control.

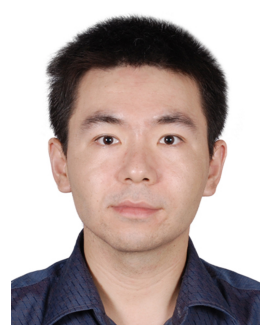

Yuming Zhao received the B.Sc. and Ph.D. degrees in Tsinghua University, Beijing, China, in 2006 and 2001, respectively, all in electrical engineering. $\mathrm{He}$ is now a Senior Engineer of Professor level in Shenzhen Power Supply Company, Shenzhen, China. His research interests include HVDC and lowvoltage DC system analysis, control and protection.

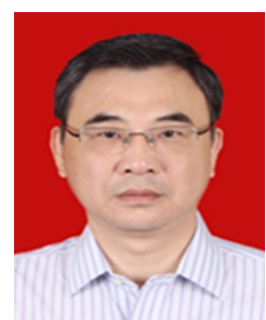

Gang Wang received the Ph.D. degree in electrical engineering and automation from TianJin University, TianJin, China, in 1998. He is currently a Professor with the School of Electric Power Engineering, South China University of Technology, Guangzhou, China. He is the Director of the Power Engineering and Technology Research and Development Center, Guangdong, China. His major research interests include power system protection and control, fault analysis, power system planning and reliability, and high-voltage direct current technology.

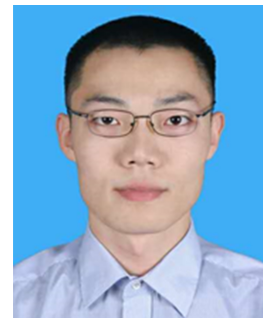

Longjun Wang received the $\mathrm{Ph} . \mathrm{D}$. degree in electrical engineering from the South China University of Technology, Guangzhou, China, in 2010. He is currently a Lecturer with the School of Electric Power Engineering, South China University of Technology. His major research interests include power system reliability and planning, and artificial intelligence application. 